\title{
Water Film in Saturated Sand
}

\author{
Xiaobing LU ${ }^{1}$, X. H. ZHANG ${ }^{1}$, Cui PENG ${ }^{2}$ \\ ${ }^{1}$ Institute of Mechanics, Chinese Academy of Sciences, Beijing, China \\ ${ }^{2}$ Institute of Mountain Hazard and Environment, Chinese Academy of Sciences, Chengdu, China \\ Email:xblu@imech.ac.cn \\ Received June 16, 2009; revised July 22, 2009; accepted August 4, 2009
}

\begin{abstract}
In order to investigate the initiation mechanism of landslide and debris flow occurring on a gentle slope. The formation and evolution of water film (or crack) in saturated sand is analyzed by numerical and theoretical simulations under given conditions. First a psudo-three-phase model is presented considering the movement of skeleton and water and the erosion. Secondly, difference method is used to analyze the formation conditions and the evolution of the velocity of water and pore pressure and porosity. Thirdly, a simplified theoretical method is presented based on the consolidation theory to analyze the initiation, expansion and close. It is shown that there are stable water films when some point is blocked and the state keeps unchangeable or there exists a thin layer with very low permeability. Once the blocked point is open, the water film will disappear gradually. The evolution of water film may be calculated by a simplified method. The analytical results are agreement well with that of Kokusho.
\end{abstract}

Keywords: Water Film, Saturated Sand, Liquefaction, Percolation, Erosion

\section{Introduction}

It is often observed that sand deposit on slope spreads laterally or even turns into landslide or debris flow, especially after earthquakes. When the deposit is multilayered, a water film will form once it is liquefied [1], which may serve as a sliding surface for the postliquefaction failure. As a result, landslide or debris flow may happen on very gentle slope. Seed [2] was the first to suggest that the existence of "water film" in sand bed is the reason of slope failures in earthquakes. Later, some researchers [3-5] performed some experiments to investigate the formation of "water film" in layered sand or in sand containing a seam of non-plastic silt. However, the mechanism of cracks or "water film" in sand with porosity distributed continuously is not very clear.

A theoretical and numerical analysis is presented in this paper. Firstly, we present a pseudo-three-phase model describing the moving of liquefied sand and give some theoretical analysis. Then we give the numerical simulations under four initial and boundary conditions. Secondly, we present a simplified method to analyze the evolution of the water film.

\section{Formulation of the Problem}

Consider a saturated horizontal sand stratum, with the porosity changing only vertically. The fine grains may be eroded from the skeleton and the eroding relation is assumed as follows [6-7]

$$
\begin{aligned}
& \frac{1}{\rho_{s}}\left(\frac{\partial Q}{\partial t}+u_{s} \frac{\partial Q}{\partial x}\right)=\frac{\lambda}{T}\left(\frac{u-u_{s}}{u^{*}}-q\right) \\
& \text { if }-\varepsilon(x, 0) \leq \frac{Q}{\rho_{s}} \leq \frac{Q_{c}(x)}{\rho_{s}}
\end{aligned}
$$

$\frac{1}{\rho_{s}}\left(\frac{\partial Q}{\partial t}+u_{s} \frac{\partial Q}{\partial x}\right) \leq 0$ otherwise, including $u=0$

where the $x$ axis is upward, $Q$ is the mass of sand eroded per unit volume of the sand/water mixture, $\rho_{s}$ is the density of the grains, $u$ and $u_{s}$ are the velocities of the percolating fluid containing fine sand particles and the sand grains, $q$ is the volume fraction of sand carried in the percolating fluid, T and $u^{*}$ are physical parameters, $\lambda$ is a small dimensionless parameter, $\varepsilon(x, t)$ is the porosity, $Q_{c}(x)$ is the maximum $Q$ that can be eroded at $\mathrm{x}$.

Considering the eroding of the fine grains, a psudothree-phase model is presented here. The mass conservation equations are: 


$$
\begin{gathered}
\frac{\partial(\varepsilon-q) \rho}{\partial t}+\frac{\partial(\varepsilon-q) \rho u}{\partial x}=0 \\
\frac{\partial q \rho_{s}}{\partial t}+\frac{\partial q \rho_{s} u}{\partial x}=\frac{\partial Q}{\partial t}+u_{s} \frac{\partial Q}{\partial x} \\
\frac{\partial(1-\varepsilon) \rho_{s}}{\partial t}+\frac{\partial(1-\varepsilon) \rho_{s} u_{s}}{\partial x}=-\frac{\partial Q}{\partial t}-u_{s} \frac{\partial Q}{\partial x}
\end{gathered}
$$

in which $\rho$ is the density of water. From these follows a general equation:

$$
\varepsilon u+(1-\varepsilon) u_{s}=U(t)
$$

in which $U(t)$ is the total mass of fluid and grains at a transect. The momentum equations may be written as

$$
\begin{aligned}
& {\left[(\varepsilon-q) \rho+q \rho_{s}\right]\left(\frac{\partial u}{\partial t}+u \frac{\partial u}{\partial x}\right)=-\varepsilon \frac{\partial p}{\partial x}-\frac{\varepsilon^{2}\left(u-u_{s}\right)}{k(\varepsilon, q)}-} \\
& {\left[(\varepsilon-q) \rho+q \rho_{s}\right] g} \\
& {\left[(\varepsilon-q) \rho+q \rho_{s}\right]\left(\frac{\partial u}{\partial t}+u \frac{\partial u}{\partial x}\right)+(1-\varepsilon) \rho_{s}\left(\frac{\partial u_{s}}{\partial t}+u_{s} \frac{\partial u_{s}}{\partial x}\right)} \\
& =-\frac{\partial p}{\partial x}-\frac{\partial \sigma_{e}}{\partial x}-\left[(\varepsilon-q) \rho+q \rho_{s}\right] g-(1-\varepsilon) \rho_{s} g \\
& \quad-\left(\frac{\partial Q}{\partial t}+u_{s} \frac{\partial Q}{\partial x}\right)\left(u-u_{s}\right)
\end{aligned}
$$

in which Equation (7) denotes the momentum conservation of grains, Equation (8) denotes the total momentum conservation, the last term on the right hand side of Equation (8) denotes the momentum caused by the eroded fine grains, $p$ is the pore pressure, $k$ is the permeability, $\sigma_{e}$ is the effective stress, $\theta$ is the slope. Here $k$ is assumed to be a function of $\varepsilon$ and $q$ in the following form

$$
k(\varepsilon, q)=k_{0} f(q, \varepsilon)=k_{0}(-\alpha q+\beta \varepsilon)
$$

in which $\alpha, \beta$ are parameters and $1<\beta<<\alpha$, we choose to let $\alpha$ much greater than $\beta$, so that changes in $\mathrm{q}$ overweighs that of $\varepsilon$.

\section{Numerical Simulations}

Based on the model presented above, we will analyze the occurrence of the crack in saturated sand, This case is about the cracks in a liquefied sand $\left(\sigma_{e}=0, \tau \approx 0\right)$ where the grains sink while the water is pressed to move upward just like the consolidation. Here, the sand column is assumed to be long enough to neglect the boundary effects. At the same time, we neglect some factors which may be important in other cases. We will simulate this problem by different methods.

Being an appropriate constant, the mass conservation
Equation (6) yield

$$
\varepsilon u+(1-\varepsilon) u_{s}=U(t)=0
$$

assuming both $\mathrm{u}$ and $\mathrm{u}_{\mathrm{s}}$ to be zero at $\mathrm{x}=0$.

Let $\mathrm{T}$ be the appropriate characteristic time in Equation (10), $\mathrm{u}_{\mathrm{t}}$ the characteristic velocity and L the characteristic length, and let

$$
\bar{u}=\frac{u}{u_{t}}, \tau=\frac{t}{T}, \xi=\frac{x}{T u_{t}}
$$

Then Equations (3) and (4) can be rewritten in the non- dimensional form:

$$
\begin{aligned}
& \frac{\partial \varepsilon}{\partial \tau}+\frac{\partial \varepsilon \bar{u}}{\partial \xi}=\bar{u} \frac{u_{t}}{u^{*}(1-\varepsilon)}-q \\
& \frac{\partial q}{\partial \tau}+\frac{\partial q \bar{u}}{\partial \xi}=\bar{u} \frac{u_{t}}{u^{*}(1-\varepsilon)}-q
\end{aligned}
$$

For $\mathrm{Tg} / \mathrm{u}_{\mathrm{t}}>>1$, the inertia terms are negligible and the last equation of Equation (8) becomes

$$
\begin{aligned}
\bar{u} & =\left(\frac{1-\varepsilon}{\varepsilon}\right)^{2}(\varepsilon-q) f(q, \varepsilon) \frac{k_{0} \rho_{s} g\left(1-\rho / \rho_{s}\right)}{u_{t}} \\
& =\left(\frac{1-\varepsilon}{\varepsilon}\right)^{2}(\varepsilon-q) f(q, \varepsilon)
\end{aligned}
$$

where $u_{t}$ is

$$
u_{t}=k_{0} \rho_{s} g\left(1-\rho / \rho_{s}\right) .
$$

Now the problem reduces to finding $\varepsilon(\xi, \tau)$ and $q(\xi, \tau)$ as solutions to Equation (12). The initial conditions are clearly

$$
\varepsilon(\xi, 0)=\varepsilon_{0}(\xi), q(\xi, 0)=0
$$

In order to de-couple the problem from the complication arising from the effect of the consolidation wave due to the bottom of the sand column, we assume that the sand column is very tall so that cracks may develop before the consolidation wave arrives.

\section{Numerical Results and Analysis}

In this section, we will solve Equation (17) by using of the finite differential method under two types of initial conditions to study the evolution of water film.

Parameters adopted in simulation are as follows(Lu et al., 2007): $\beta=47 \sim 56, \quad \rho_{s}=2400 \mathrm{~kg} / \mathrm{m}^{3}, \quad \rho_{w}=1000 \mathrm{~kg} / \mathrm{m}^{3}$, $u^{*}=0.04, k_{0}=4 \times 10^{-6} \mathrm{~m} / \mathrm{s}, \alpha=1, \kappa=50.0, a=0.08$, time step $\Delta \tau=9 \times 10^{-4}$, step length $\Delta x=0.01$, critical pore pressure $P_{c r}=0.25 \mathrm{MPa}, L$ is the length of the sand column. 
The results are given as follows:

1) Condition 1: The initial porosity changes continuously, and assuming that once a place is choked, the choked state keeps unchangeable.

It is shown from Figure 1 that when some position is choked, the porosity just below this position increases gradually till it equals 1.0 . We think there is a water film full of water occurring here. Once it occurs, the water film expands gradually.

Figure 2 shows the velocity development of pore water. It is shown that the velocity of pore water first increases because of the high hydraulic gradient. Nevertheless, with the decrease of porosity and the permeability, the velocity decrease to near zero. Outside the water film, the velocity of pore water changes little. We may think there is discontinuity exists between inside and outside the water film.

Figure 3 shows the development of the fine grains eroded from the skeleton. It is shown that at the choked position, the sum of fine grains decreases fast because of the small velocity difference between the pore water and grains, while above the choked position, the sum of fine grains increases fast. The eroded fine grains are related with the velocity difference between pore water and

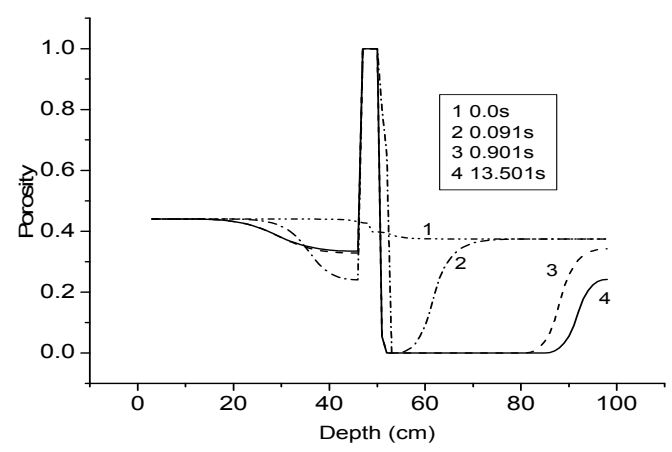

Figure 1. Development of porosity under assumption 1.

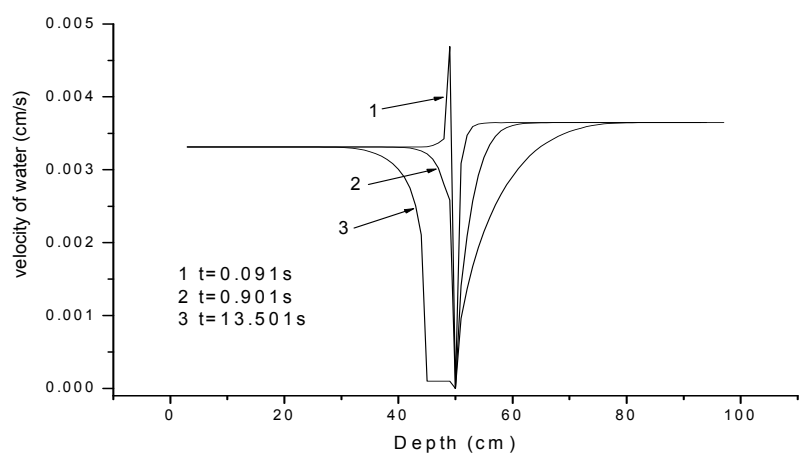

Figure 2. Development of velocity of pore water under assumption 1.

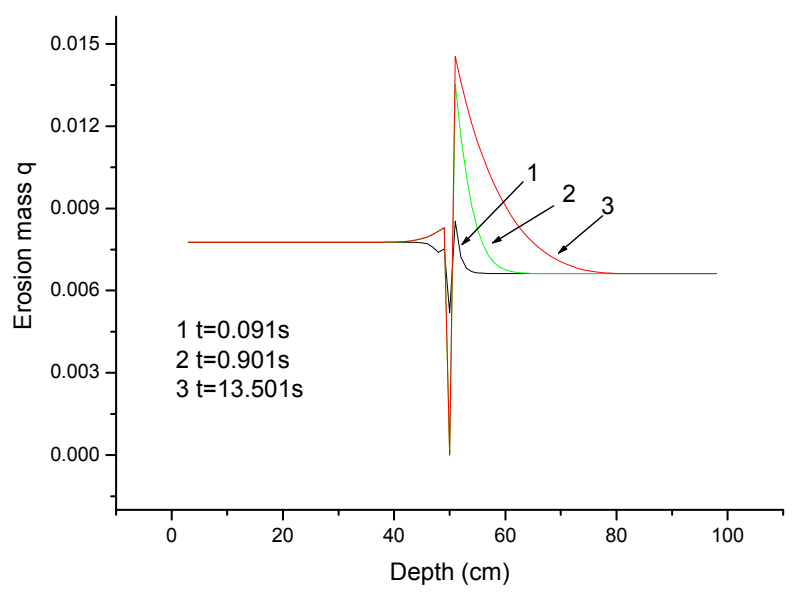

Figure 3. Development of $q$ under assumption 1.

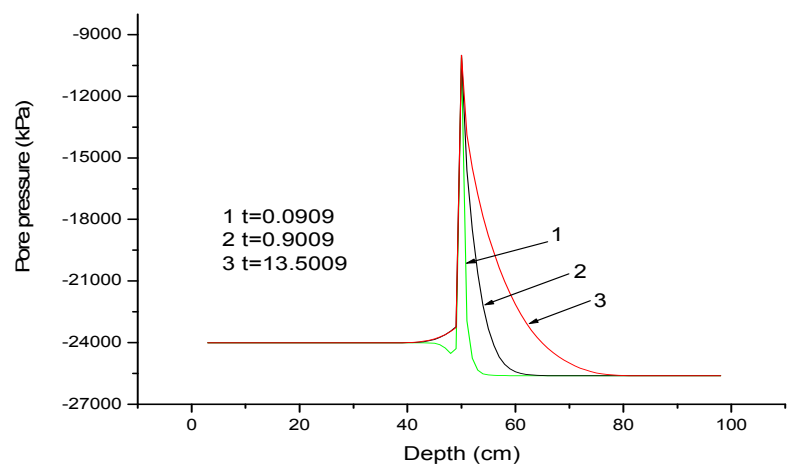

Figure 4. Development of pore pressure under assumption 1 ( minus denotes compressive pore pressure).

grains according to Equations (1) and (2). Therefore, in the water film, the eroded fine gains $q$ is very small because the velocity difference is near zero, while it is big above the water film because the velocity difference is big. Some distance away from the water film, the eroded fine grains is very small and does not change with position.

Figure 4 shows the distribution of pore pressure. It can be seen that the pore pressure is discontinuity. The peak of the pore pressure is at the choked point. It causes the pore water percolate upward and the porosity above the choked point increase. The pore pressure below the choked position becomes from the initial continuous to increase suddenly. The reason is that the chocked position leads the pore water flow difficult and thus causes the pore pressure increase.

The reason may be explained as follows: when the sand column is choked, the velocity of pore water decreases to near zero and a high hydraulic gradient forms at this position because the porosity is very small. Thus the discontinuity of the pore water velocity, grain velocity and the amount of fine grains eroded from the skele- 
ton occurs. The pore water beneath the choked position flows upwards but passes through the choked position very difficult, which leads a net inflow of pore water just beneath the choked position. Therefore, water film forms gradually at this position. With the pore water beneath the choked position flows upwards while the grains sink gradually, the water film becomes wider and wider. The expanding velocity of water film is equal to that of the discontinuity

$$
D=\left\lfloor\left(1-\varepsilon^{+}\right) u_{s}^{+}-\left(1-\varepsilon^{-}\right) u_{s}^{-}|/|\left(1-\varepsilon^{+}\right)-\left(1-\varepsilon^{-}\right)\right]=u_{s}^{-},
$$

where $u_{s}^{+}, \varepsilon^{+}$denotes the velocity of grains and porosity in the water film and thus $u_{s}^{+}=0, \varepsilon^{+}=1, u_{s}^{-}, \varepsilon^{-}$ denotes the velocity of grains and porosity beneath the water film. It can be seen that the expanding velocity of water film is the same as the settlement velocity of grains. This velocity will change with the other parameters of the sand column.

2) Condition 2: The porosity distribution is the same as that in Condition 1, but there is no choking-statekeeping assumption.

It is shown from Figure 5 that when some position is choked, the porosity at this position increases gradually till water film occurs. If the choked position is dredged again when the pore pressure is over the critical pore pressure, the pore pressure at the chocked position decreases gradually, and the pore water and grain velocities becomes smooth from discontinuity states. At last, water film disappears.

Figure 6 gives the development of velocity of pore water under Condition 2. It is shown that the velocity of pore water develops to be discontinuity at the first stage after water film occurring. When the choked position is dredged, the velocity of pore water below the chocked position increases while the velocity in water film is still zero. The increase may be that the pore water flows through the once chocked position and the pore pressure decreases gradually. At last, the velocity of pore water becomes smoothly.

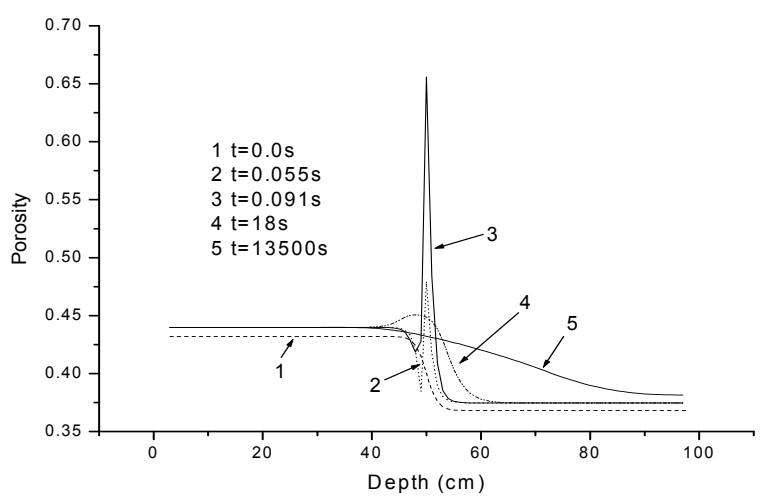

Figure 5. Development of porosity under assumption 2.

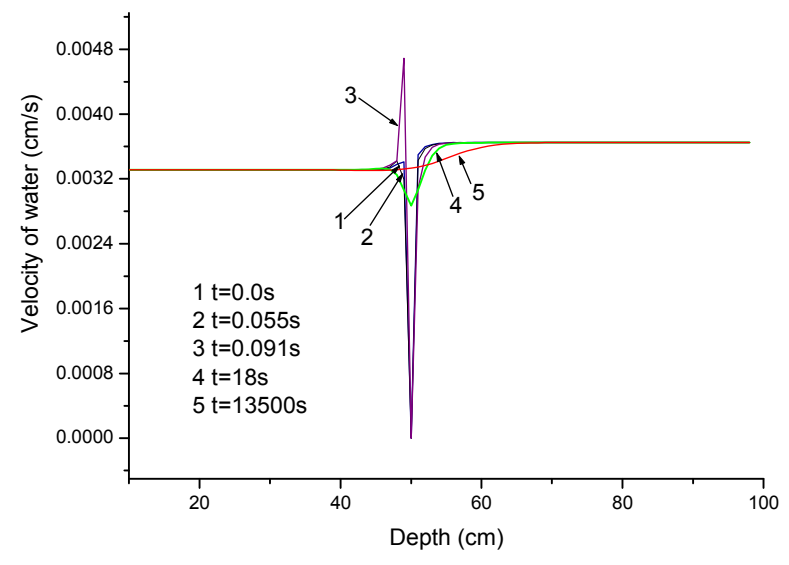

Figure 6. Development of velocity of pore water under assumption 2.

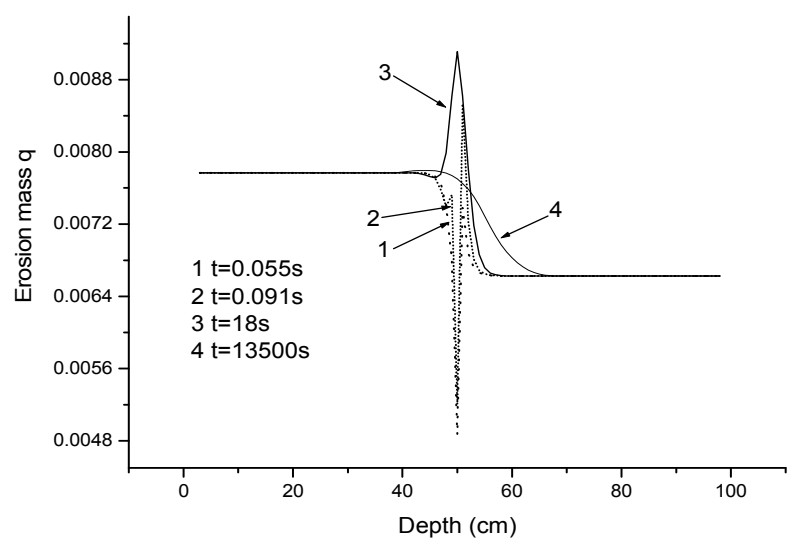

Figure 7. Development of $q$ under assumption 2.

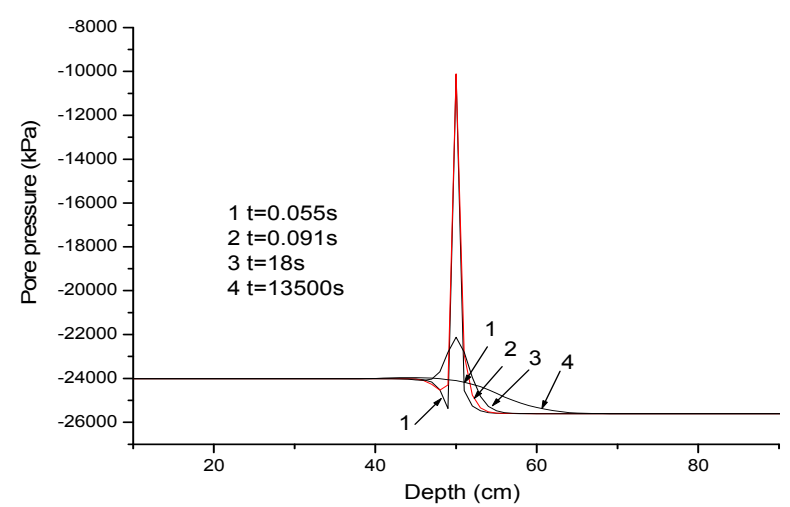

Figure 8. Development of pore pressure under assumption 2.

Figure 7 gives the distribution and development of the eroded fine grains under Condition 2. It is shown that the distribution of eroded fine grains is sharply decreased in the water film, but with the disappearing of water film, the velocity of pore water increases first and then de- 
creases, so the eroded fine grains increase first at the position once being water film, and then the distribution of eroded fine grains becomes smooth.

Figure 8 gives the development of pore pressure under Condition 2. It is shown that the development of pore pressure increases fast once the chocked position forms. Nevertheless, with the dredging, the water film disappears gradually and the pore pressure decreases gradually.

At the first stage, the water film forms similar to that under Condition 1. Nevertheless, once the pore pressure is over the critical value, water can flow through the choked position again. The grains above the choked position catch up the grains beneath the choked position. The pore pressure becomes smoothly gradually and the water film disappears.

\section{A Simplified Evaluation Method}

Although the numerical simulation may give more information about the evolution of the water film, a simplified method is needed. Florin et al. [8] pointed out that when the settling particles reach solid material, which is usually the non-liquefied underlying soil, or the container base in an experiment, they accumulate to form a solidified zone which increases in thickness with time. A solidification front therefore moves upward until it reaches the surface or the overlying non-liquefied material. Zhang et al. [5] and Scott et al. [9] had analyzed the development of the solidification. Here we present a simplified analytical method of the water film in saturated sand with initial non-uniform grade series.

Assuming that the whole mass reaches its terminal velocity, $k$, which is the permeability, instantaneously at the end of liquefaction, Florin gave an expression for the constant velocity, $\dot{z}$, of the solidification front:

$$
\dot{z}=\frac{\rho}{\rho_{w}} \frac{1-n_{1}}{n_{2}-n_{1}} k
$$

in which $\rho^{\prime}=\rho_{s}-\rho_{w}$ is the buoyant unit weight of the liquefied soil, $n_{1}$ is the porosity of the liquefied soil, $n_{2}$ is the porosity of the solidified soil.

From Equation (16), we can obtain the duration of liquefaction and subsequent excess pore pressure decline for any point in the soil column.

$$
t=\frac{\rho_{w}}{\rho} \frac{n_{2}-n_{1}}{1-n_{1}} \frac{h}{k}
$$

$h$ is the height of any point in the soil column.

The final settlement of the top surface of the sand layer is

$$
\Delta L=\frac{n_{0}-n_{1}}{1+n_{0}} H
$$

This occurs at time given by Equation (17), so the rate of settlement is

$$
\dot{s}_{a}=\frac{\gamma^{\prime} k}{\gamma_{w}}
$$

The settlement at any time is

$$
s_{a}=\frac{\gamma^{\prime} k}{\gamma_{w}} t
$$

The settlement velocity $v_{e}$ of the elements above the water film is determined by the combined permeability $k_{e s}$ of the middle layer and the upper layer [10]:

$$
k_{e s}=1 / \sum_{1}^{m} L_{i}^{m} / k_{i}
$$

The upward seepage flow has the same velocity:

$$
v=k_{e s} i_{e}
$$

$i_{e}$ is the average hydraulic gradient.

In fact, the particles and solidified soil are compressible, the skeleton may consolidate by the geostatic stress after the solidification. If the permeability is small, the excess pore pressure does not disperse immediately with the increase of solidification zone. The deform of the skeleton of the sand by the geostatic stress in the solidification zone may be expressed as [5]

$$
s_{2}=\frac{1}{2} \frac{\rho g}{m_{s}} x^{2}(t)
$$

in which the compressible modulus $m_{s}$ is assumed as a constant. The percolation is assumed to obey the Darcy lay. The total deformation is:

$$
\Delta s=\Delta s_{1}+\Delta s_{2}
$$

Instituting Equations (16) and (23) into (24), it yields

$$
\Delta s=\frac{n_{20}-n_{21}}{1-n_{20}}(\Delta z+\Delta s)+\frac{\dot{\rho} g}{m_{s}} z \Delta z
$$

The settling velocity of the surface is

$$
\frac{\Delta s}{\Delta t}=k \frac{\rho}{\rho_{w}}
$$

The increase velocity of the thickness of solidification is 


$$
\frac{\Delta z}{\Delta t}=\frac{k \rho^{\prime}}{\rho_{w}} /\left(\frac{n_{0}-n_{1}}{1-n_{1}}+\frac{1-n_{1}}{1-n_{0}} \frac{\rho^{\prime} g}{m_{s}} z\right)
$$

The time for the solidification front to arrive at any location is

$$
t=\frac{\rho_{w}}{k \rho^{\prime}}\left(\frac{n_{0}-n_{1}}{1-n_{1}} z+\frac{1}{2} \frac{1-n_{1}}{1-n_{0}} \frac{\rho^{\prime} g}{m_{s}} z^{2}\right)
$$

The side friction, if influential in the situation, may be expressed as

$$
\sigma_{s}=\mu K_{0} \sigma_{z}
$$

This effect should be considered in the pore-pressuregradient.

The effect of the changes of porosity on the permeability is considered here using a linear relation:

$$
k=k_{0}\left[1-\alpha\left(n_{0}-n\right)\right]
$$

in which $k_{0}$ is the initial porosity, $\alpha$ is a parameter, $n_{0}$ is the initial porosity, $k$ is the permeability when the porosity is $n$.

Thus, by considering Equations (29) and (30) in the pore pressure gradient and the consolidation of the solidification zone, we can compute the course that the water film increases and closes.

\section{Comparison with the Experimental Results}

The results are compared with the experimental data of Kokusho [10] (Figure 5). In Kokusho's experiment, a saturated loose sand layer of $200 \mathrm{~cm}$ depth sandwiches a seam of nonplastic silt in the middle $(96 \mathrm{~cm}$ from the bottom). The initial void ratios of the upper, the middle and the lower sand layers are $0.924,1.5$ and 0.831 , respectively; and the initial permeability coefficients are $0.04 \mathrm{~cm} / \mathrm{s}, 0.00018 \mathrm{~cm} / \mathrm{s}$ and $0.04 \mathrm{~cm} / \mathrm{s}$, respectively. The silt seam is about $4 \mathrm{~mm}$ thick. The saturated sand is in a tube with an inner diameter of $13 \mathrm{~cm}$ and a height of $211.5 \mathrm{~cm}$. The one-dimensional sand layer is instantaneously liquefied under a loading caused by a steel hammer. In the computation for comparison, we adopted the data in Kokusho [10] (Table 1). In Figure 9, we can see that the two results are agreement with each other in some extent.

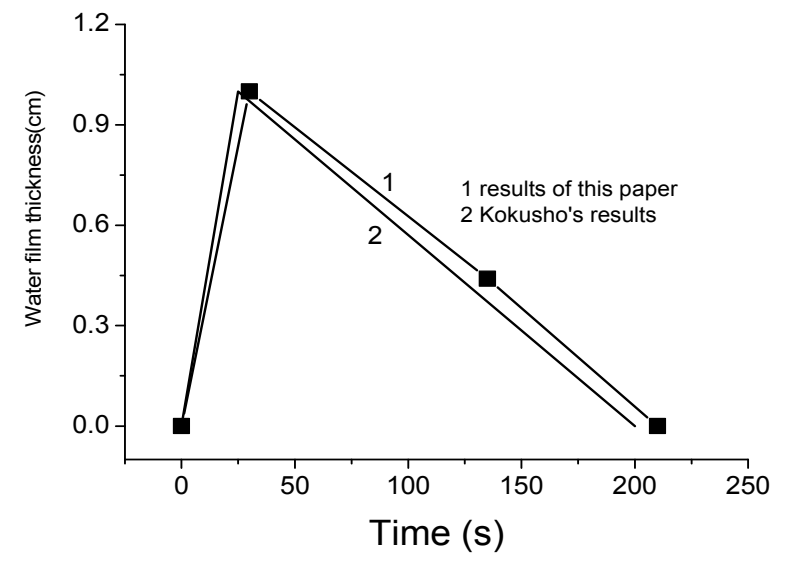

Figure 9. The comparison of our results with the experimental of Kokusho [10].

\section{Conclusions}

Numerical simulations under four conditions have shown that the stable water films occur only in the conditions that: (1) the porosity of the upper part of the sand column is smaller than that of the lower, and (2) the keeping of the jamming state or the effective stress to prevent the free dropping of the grain or the skin friction in Kokusho's experiments is needed. A simplified method for evaluating the thickness of water film is presented and the computed results are close to the experimental results.

Although some main characteristics of the formation of water film are obtained based on the presented model, some improvements such as the erosion relation Equation (1) and the permeability function Equation (9) are needed in order to make the model more practical. The analyze on the evolution of the sand column in two and three dimensions will be processed in the future.

\section{Acknowledgements}

This paper is supported by National Basic Research Program of China "Activity characteristics and formation rules of secondary mountain hazard of earthquake" (No. 2008CB425802) and Key Program of Chinese Academy of Sciences (No. KZCX2-YW-302-02).

Table 1. The data of the parameters.

\begin{tabular}{cccccc}
\hline sand & Thickness $(\mathrm{cm})$ & Relative density $(\%)$ & Permeability coefficient (cm/s) & Initial pore ratio & The max. strain (\%) \\
\hline The upper layer of sand & 103.6 & 14 & 0.04 & $0.924(0.48)$ \\
seam & 0.4 & & $1.8 \mathrm{E}-4$ & 2.4 & $1.5(0.6)$ \\
The lower layer of sand & 96 & 39 & 0.04 & $0.831(0.454)$ \\
\hline
\end{tabular}




\section{References}

[1] T. Kokusho, K. Watanabe, and T. Sawano, "Effect of water film on lateral flow failure of liquefied sand," Proceedings in $11^{\text {th }}$ European Conference on Earthquake Engineering., Paris, CD publication, ECEE/T2/kokeow.pdf, 1998.

[2] H. B. Seed, "Design problems in sand liquefaction," Journal of Geotechnical Engineering, ASCE, Vol. 113, No. 8, pp. 827-845, August 1987.

[3] G. L. Fiegel and B. L. Kutter, "Liquefaction mechanism for layered sands," Journal of Geotechnical Engineering., ASCE, Vol. 120, No. 4, pp. 737-755, Spring 1994.

[4] T. Kokusho, "Water film in liquefield sand and its effect on lateral spread," Journal of Geotechnical and Geoenvironmental Engineering, Vol. 125, No. 10, pp. 817-826, October 1999.

[5] J. F. Zhang, "Experimental study on the strengthening of percolation and the damage of structure under impact loading," Dissertation for Ph. D, Institute of Mechanics, Chinese Academy of Sciences, 1998.

[6] C. M. Cheng, Q. M. Tan, and F. J. Peng, "On the mechanism of the formation of horizontal cracks in a vertical column of saturated sand," ACTA Mechanica Sinica (English Serials), Vol. 17, No. 1, pp. 1-9, March 2000.

[7] X. B. Lu and Z. M. Zheng, "Formation of water film in saturated sand," ACTA Mechania Sinica, Vol. 22, No.3, pp. 377-383, June 2006.

[8] V. A. Florin and P. L. Ivanov, "Liquefaction of saturated sandy soils," Proceedings in $5^{\text {th }}$ International Conference on Soil Mechanic Foundation Engineering, Paris, 1, pp. 107-111, 1961.

[9] R. F. Scott, "Solidification and consolidation of a liquefied sand column," Soils and Foundations, Vol. 26, No. 4, pp. 23-31, 1986.

[10] T. Kokusho and T. Kojima, "Mechanism for postliquefaction water film generation in layered sand," Journal of Geotechnical and Geoenvironmental Engineering, ASCE, Vol. 128, No. 2, pp. 129-137, February 2002. 